\title{
INTERDIFFUSION OF Mo AND W UNDER IN-PILE IRRADIATION CONDITIONS
}

\author{
A.S. Ivanov ${ }^{1}$, V.A. Churin ${ }^{2}$ \\ NRC "Kurchatov Institute", \\ Academician Kurchatov Square 1, Moscow 123182 Russia \\ ${ }^{1}$ asi.kiae@gmail.com, ${ }^{2}$ Churin_VA@nrcki.ru
}

\begin{abstract}
The study has established that reactor radiation leads to a significant increase of interdiffusion coefficients in a $M o-W$ alloy compared to thermally activated diffusion at temperatures of $\sim 1500^{\circ} \mathrm{C}$. Furthermore, a weakening of their concentration dependence is observed. The approximation procedure developed in this work allowed approximating experimental data for each sample using variation of only one parameter. It has been demonstrated that the interaction of molybdenum and tungsten nuclei with fast neutrons as well as with hard gamma quanta may cause the observed diffusion effects.
\end{abstract}

KEYWORDS: Interdiffusion, fuel cladding, irradiation, neutron flux, photoeffect, Compton effect, electron-positron pair production.

\section{INTRODUCTION}

Investigation of the interdiffusion in the binary $M o-W$ system received attention of a number of authors due to the wide application of this pair in various technical areas [1-3]. The studies included such aspects as temperature dependencies of concentration profiles, diffusion coefficients as a function of grain size, and the contribution of diffusive transfer occurring along the grain boundaries. Based on extensive experimental data, the following phenomenological relationship for determining the diffusion coefficient $D$ as a function of temperature and concentration of components was derived [1].

$$
D=0,11 \exp \left(3,45 C_{M o}\right) \exp \left(-\frac{126000}{R T}\right),
$$

where $C_{M o}$ is molybdenum concentration, $R$ is the universal gas constant, and $T$ is temperature. Here and elsewhere the diffusion coefficient is measured in $\mathrm{cm}^{2} / \mathrm{s}$.

The molybdenum concentration dependence $C_{M o}(\zeta)$ presented as a function of a known reduced variable [1]

$$
\zeta=\frac{x}{2 \sqrt{t}}\left[0,11 \exp \left(-\frac{126000}{R T}\right)\right]^{-\frac{1}{2}}
$$

allowed describing a large body of experimental data. Therefore, the $M o-W$ pair can be considered as a basic model system for studying the impact of external factors on diffusive transfer processes in solids. One 
of such external factors is the effect of reactor radiation. For the relatively low-temperature range, the effect of reactor radiation on diffusion processes is well known [4]. In experiments this effect manifests itself in acceleration of diffusion (radiation-enhanced diffusion) where the diffusion coefficients may increase by several orders of magnitude. Various mechanisms are used to explain this phenomenon, for example, a mechanism of diffusion acceleration resulting from "radiation shaking" of solids [5], or a crowdion mechanism of diffusion acceleration due to displacement of atom groups [6].

It has been demonstrated that the radiation-enhanced diffusion plays a predominant role at temperatures below $1200^{\circ} \mathrm{C}$ compared to the thermally activated diffusion owing to generation of nonequilibrium vacancies in irradiated materials. However, it is believed [7] that at elevated temperatures the role of reactor radiation in diffusion processes is cancelled out, with the thermally activated diffusion making the major contribution to atom transfer. The effect of the technology used for applying the tungsten coating onto the molybdenum cladding of a fuel element on the diffusive transfer in the binary $M o-W$ system under irradiation was discussed in [2]. Also there were presented results suggesting that reactor radiation may have effect on the diffusive transfer at temperatures significantly higher than $1200^{\circ} \mathrm{C}$.

The purpose of this work is to carry out a comparative analysis of results of reactor experiments for studying the interdiffusion of molybdenum and tungsten, and to evaluate the effect of reactor radiation on concentration dependences of diffusion coefficients.

\section{DESCRIPTION OF THE EXPERIMENT}

\subsection{Test conditions and samples under study}

The analysis was performed for results of experimental studies of samples of fuel element claddings that passed in-pile tests as part of the ENISSEY prototype nuclear power system [3]. Fuel element claddings in this system served as emitters of thermionic fuel elements (TFE) and represented a bimetallic system comprised of a cylindrical molybdenum tube with a diameter of $\sim 18 \mathrm{~mm}$ and a wall thickness of $1 \mathrm{~mm}$, with $\sim 100 \mu \mathrm{m}$ thick tungsten coating applied on the outside. The paper presents results of the studies of nine samples taken from claddings located in various parts of the reactor core.

For the studies, about 5-mm wide annular pieces were cut out of the cylindrical TFE claddings. The samples were cut out in a special hot cell designed for handling radioactive materials. After that the samples passed through a complicated and time-consuming preparation process, including grinding, polishing and washing to remove process and radioactive contaminations.

The sample irradiation times and temperatures are presented in Table 1 below.

Table 1. Sample irradiation times and temperatures.

\begin{tabular}{|l|l|c|c|c|c|c|c|c|c|}
\hline Sample & 1 & 2 & 3 & 4 & 5 & 6 & 7 & 8 & 9 \\
\hline$t, \mathrm{~h}$ & 12350 & 3000 & 3000 & 3900 & 3900 & 4828 & 3000 & 3900 & 4828 \\
\hline$T^{\circ} \mathrm{C}$ & 1550 & 1540 & 1590 & 1550 & 1500 & 1590 & 1470 & 1580 & 1530 \\
\hline
\end{tabular}

\subsection{Measurement procedure}

The interdiffusion of tungsten and molybdenum was studied using the MAR-2 commercial X-ray spectrum analyzer [2] provided with wavelength spectrometers. The analyzer has two spectrometers with horizontal movement of crystals. An electron gun with a V-shaped tungsten cathode is used for excitation of characteristic X-ray radiation in the sample. Highly stable voltage $\mathrm{E}=30 \mathrm{kV}$ was supplied to the gun cathode. The electron beam generated by the gun at the current of $\sim 10^{-7} \mathrm{~A}$ was focused by electron lenses onto the target area of the cladding placed on the sample chamber stage, and evacuated to a high vacuum. The electron beam diameter was $\sim 1 \mu \mathrm{m}$. Intensities of the characteristic X-ray radiation of $M o_{K \alpha l}$ and $W_{L \alpha l}$ spectral lines were recorded at the count rate $\mathrm{N} \leq 10^{4}$ pulses/sec by proportional counters, and measured with the spectrometer recording system. 
The samples were delivered to the analyzer in lead shielded containers and placed into a specially designed sample stage allowing the samples to be rotated and moved at the speed of up to $9 \cdot 10^{-4} \mathrm{~m} / \mathrm{h}$. When recording the characteristic X-ray radiation intensities, an area to be analyzed was selected using a microscope at the magnification power of 500 and resolution of $\sim 10^{-5} \mathrm{~m}$.

The studies with the MAR-2 analyzer resulted in obtaining a graphical record of intensities of characteristic $\mathrm{X}$-ray radiation of $M o_{K \alpha l}$ and $W_{L \alpha l}$ spectral lines.

\section{PROCESSING AND ANALYSIS OF EXPERIMENTAL DATA}

For the MAR-2 microanalyzer, the relationship between the characteristic X-ray radiation intensity and $W$ concentration is near linear. It should be noted that for the $W$-Mo pair under study there is very little difference between atomic and volume concentrations of the components. According to our estimates, the absorption and fluorescence correction (PAP correction [8]) when determining $W$ and $M o$ concentrations in the MAR-2 microanalyzer is no greater than 3\%. However, as demonstrated in [1], the departure from linearity in the relationship under consideration may prove to be considerably greater in case of molybdenum X-ray lines. For this reason, the experimental data obtained for the tungsten line $L_{\alpha l}$ were used as a basis in our work. Volume fractions of tungsten $\left(C_{W}\right)$ in the interdiffusion region were calculated from the measured intensities of characteristic X-ray radiation from the claddings and reference samples of pure $W$ using the PAP correction. Volume fractions of molybdenum $\left(C_{M o}\right)$ were obtained from the experimental data for tungsten using the relationship $C_{M o}=1-C_{W}$. Figure 1 graphically presents distributions of the volume fraction of molybdenum in the diffusion zone for all nine examined samples.
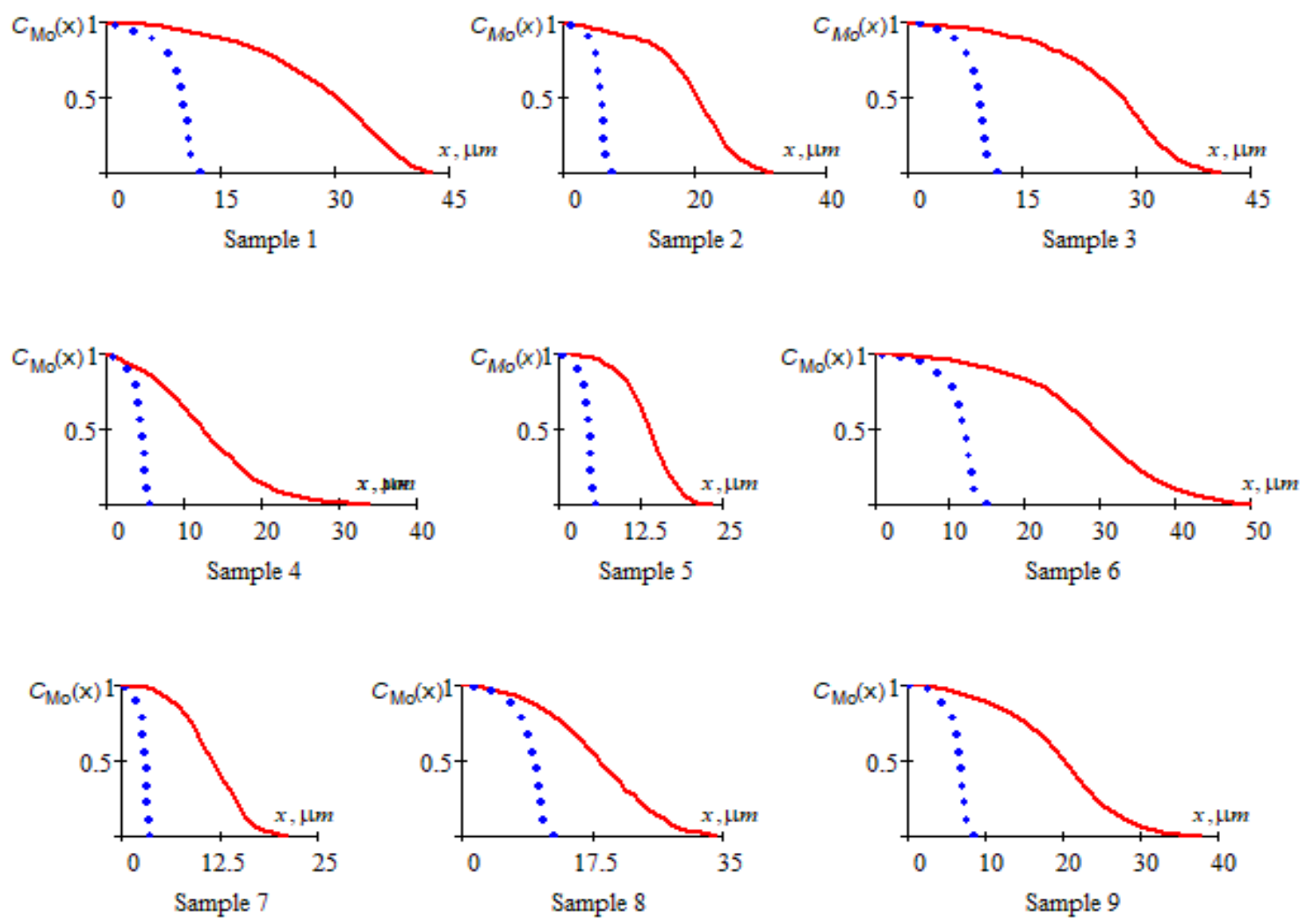

Figure 1. Distribution of volume fractions of molybdenum in the diffusion zone of each sample. 
Solid curves correspond to results obtained in reactor experiments, and dotted ones show distributions of volume fractions of molybdenum for thermally activated diffusion at the same temperatures and times. These curves were plotted using the $C_{M o}(\zeta)$ dependence derived from the solution of the diffusion equation [1].

According to the presented results, a significant acceleration of diffusive transfer processes takes place under conditions of the reactor core at temperatures greater than or equal to $1500^{\circ} \mathrm{C}$. There is observed a several-fold increase in the characterstic diffusion length compared to the thermally activated diffusion at the same temperatures and times. Hence conclusions that at high temperatures the effect of reactor radiation on diffusive transfer is negated are incorrect. Thus we see the need to identify other mechanisms that would allow explaining why the diffusive transfer accelerates under the reactor core conditions.

\section{CONCENTRATION DEPENDENCE OF THE DIFFUSION COEFFICIENT}

Let us now proceed with studying the concentration dependence of the diffusion coefficient. The interdiffusion coefficients $D(C)$ were calculated from the dependences of $M o$ or $W$ element concentration on distance in the diffusion zone by the Boltzmann-Matano method [1,9]. In this method the concentration dependence of the diffusion coefficient is defined by the following relationship:

$$
D\left(C^{\prime}\right)=-\frac{1}{2 t}\left(\frac{d x}{d C}\right)_{C^{\prime}} \int_{0}^{C^{\prime}} x d C
$$

where $t$ is the diffusion time, and $x$ is the coordinate on the $C(x)$ graph. Derivatives and integrals in the relationship (3) are found from concentration profiles obtained in the experiments (see, for example, [9]). Dots $(\bullet)$ in Figure 2 represent common logarithms of diffusion coefficients $\log D\left(C_{M o}\right)$ calculated from our experimental data at the volume fraction of molybdenum of $\{0,1 ; 0,2 ; \ldots 0,8\}$. Visually these points lie almost on a straight line practically for all of the studied samples. For comparison, straight lines are drawn in each of the graphs, which correspond to common logarithms of molybdenum interdiffusion coefficients in the Mo-W system and were plotted using the formula (1) for the same temperature. These straight lines describe the concentration dependence of the logarithm of thermally activated diffusion coefficient and correspond to the relationship (1). It can be seen from all of the presented graphs that, firstly, the diffusion coefficients are much greater for the in-pile irradiation conditions than for the thermally activated diffusion. This agrees well with the concentration profiles shown in Figure 1. Secondly, the molybdenum concentration dependence of the diffusion coefficient weakens under the in-pile irradiation conditions, i.e. the slope of the imaginary straight line drawn through the experimental points decreases for all of the studied samples. The change in the slope of the concentration dependence suggests that the mechanism of the reactor radiation effect is likely to be sensitive to a difference in characteristics of diffusing atoms. Probably, in this case reactor radiation has an effect not on the medium wherein atoms diffuse on the whole, but on individual atoms or their nuclei, for example, by transferring additional momentum in fast neutron and hard gamma quantum scattering processes. There is almost a two-fold difference in charge and mass of tungsten and molybdenum nuclei. The question is: What interaction mechanisms may have a significant effect in this case? For approximation of the concentration dependence of the diffusion coefficient under the in-pile irradiation conditions we introduce a function $\Delta\left(C_{M_{0}}\right)$ that is linear in concentration. In this case

$$
D\left(C_{M o}\right) \sim \exp \left\{\Delta\left(C_{M o}\right)+3,45 C_{M o}\right\} .
$$

Obviously, the function $\Delta\left(C_{M o}\right)$ shall take into account the intensity of processes of interaction of molybdenum and tungsten atoms with reactor radiation in accordance with concentrations of the alloy components. 

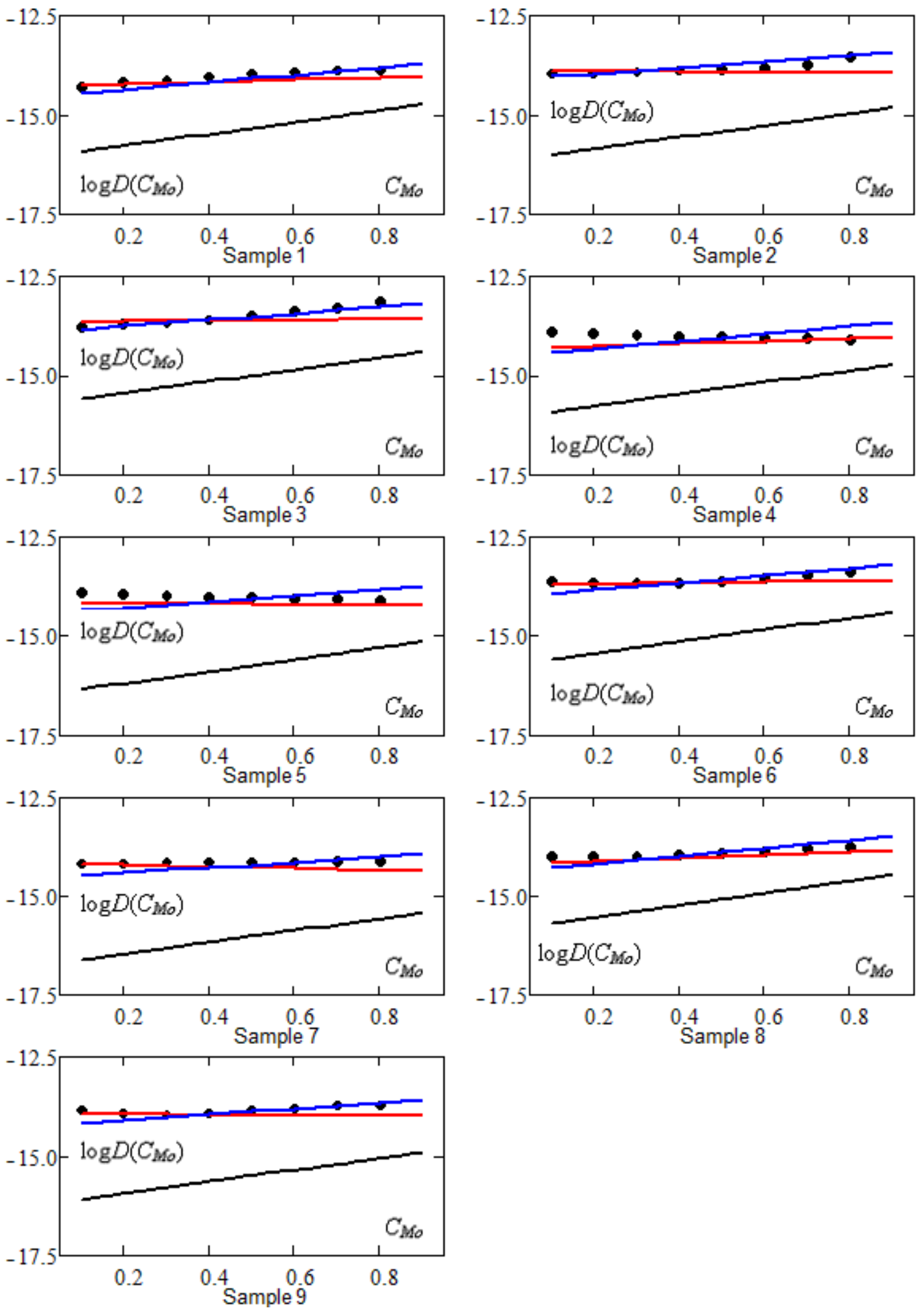

Figure 2. The lower line in each plot corresponds to the calculation of logarithms of the coefficients of thermally activated diffusion from the formula (1). The blue line was obtained by calculation from 
the formula (6) that takes into account the interaction of the alloy components with fast neutrons. The red line was obtained by calculation from the formula (7) that takes into account the interaction with hard gamma quanta. The dots in the figure correspond to graphical calculation of logarithms of the diffusion coefficients from our experimental data using the Boltzmann-Matano method [9].

The intensity of one process or another is proportional to the interaction cross-section and concentration of the respective component, therefore, the function $\Delta\left(C_{M o}\right)$ can be written as

$$
\Delta\left(C_{M o}\right) \sim\left\{C_{M o} \sigma_{M o}+\left(1-C_{M o}\right) \sigma_{W}\right\},
$$

where $\sigma_{M o}$ is the cross-section for the interaction of radiation with molybdenum atoms, and $\sigma_{W}$ is the cross-section for the interaction of radiation with tungsten atoms. It should be noted that the process in question will have a noticeable effect on the concentration dependence if cross sections of interaction with tungsten and molybdenum atoms differ considerably from each other. Here we will consider the interaction of the atoms with fast neutrons and hard gamma quanta. In case of elastic interaction between nuclei and fast neutrons, the interaction cross-section is proportional to $A^{2 / 3}$, where $A$ is the mass number of the element. Accordingly, the function $\Delta_{\mathrm{n}}\left(C_{M o}\right)$ can be written as

$$
\Delta_{n}\left(C_{M o}\right)=R_{n}\left\{C_{M o}\left(A_{M o}\right)^{2 / 3}+\left(1-C_{M o}\right)\left(A_{W}\right)^{2 / 3}\right\},
$$

where $R_{n}$ is a constant that shall be defined through the approximation of the experimental data. For the $M o-W$ pair, the ratio between the interaction cross-sections is $\sim\left(A_{M o} / A_{W}\right)^{2 / 3} \cong 0,65$.

The ability of gamma quanta to produce defects in a solid body affecting the diffusive transfer is low as compared to fast neutrons. However, this kind of radiation is present in a nuclear reactor core, and its intensity is comparable with that of the neutron flux. Since the mechanism of the effect of reactor radiation on the behavior of the diffusion coefficient at high temperatures is not completely understood, we will consider the possible effect of hard gamma radiation. As a rule, the following three kinds of processes that may result in atomic displacements are considered for the interaction between gamma quanta and metals: photoeffect, Compton effect, and electron-positron pair production within the atomic nuclei field. These processes produce high-energy electrons that can cause the formation of defects [10]. The interaction crosssection of high-energy electrons as well as the electron-positron pair production cross-section is $\sim Z^{2}$, where $Z$ is the charge number. Hence the approximation function $\Delta_{\gamma}\left(C_{M o}\right)$ may be expressed as follows:

$$
\Delta_{\gamma}\left(C_{M o}\right)=R_{\gamma}\left\{C_{M o}\left(Z_{M o}\right)^{2}+\left(1-C_{M o}\right)\left(Z_{W}\right)^{2}\right\}
$$

where $R_{\gamma}$ is the approximation constant. For the $M o-W$ pair, $\left(Z_{M o} / Z_{W}\right)^{2} \cong 0,32$.

Figure 2 shows the linear approximation of the experimental points for each of the samples (blue and red lines), which was obtained using relationship (6) for the interaction of molybdenum and tungsten nuclei with the fast neutron flux (blue line) and relationship (7) for the interaction with the gamma quanta (red line). The experimental points were approximated by the method of least squares. In each case only one fitting parameter was used for plotting the approximation line. The determined values of $R_{\gamma}$ и $R_{n}$ are presented in Table 2 below.

Table 2. Values of $R_{\gamma}$ and $R_{n}$ for the nine samples.

\begin{tabular}{|c|c|c|c|c|c|c|c|c|c|}
\hline Sample & 1 & 2 & 3 & 4 & 5 & 6 & 7 & 8 & 9 \\
\hline$R_{\gamma} \cdot 10^{4}$ & 3.3 & 4.2 & 3.8 & 3.2 & 4.3 & 3.7 & 4.8 & 3.0 & 4.2 \\
\hline$R_{n} \cdot 10^{2}$ & 4.7 & 6.3 & 5.6 & 4.8 & 6.4 & 5.4 & 6.8 & 4.5 & 6.1 \\
\hline
\end{tabular}


According to the obtained results, the experimental points much better fit the straight lines corresponding to the interaction with neutrons for the first three samples. In other six cases, however, the best result is obtained when the lines corresponding to gamma radiation are used. It is likely that the effect of both kinds of radiation takes place in each case, and if their combination is taken into account, even a better fit of the approximation line to the experimental points could be achieved.

\section{CONCLUSIONS}

Previously, it has been demonstrated that the radiation-enhanced diffusion plays a predominant role at temperatures below $1200^{\circ} \mathrm{C}$ compared to the thermally activated diffusion owing to generation of nonequilibrium vacancies in irradiated materials. However, it is believed that at elevated temperatures the role of reactor radiation in diffusion processes is cancelled out, with the thermally activated diffusion making the major contribution to atom transfer.

Results of the performed studies show that reactor radiation leads to a significant increase of the interdiffusion coefficients in the $M o-W$ alloy compared to the thermally activated diffusion at temperatures of $\sim 1500^{\circ} \mathrm{C}$. Besides, weakening of their concentration dependence is observed.

The approximation procedure developed in this work allowed approximating experimental data obtained for each sample through variation of only one parameter.

A comparative analysis of results of thermal calculations and reactor experiments for studying the interdiffusion of molybdenum and tungsten was carried out, and the effect of neutron radiation and hard gamma radiation on concentration dependences of diffusion coefficients was evaluated. It has been demonstrated that the interaction of molybdenum and tungsten nuclei with fast neutrons as well as with hard gamma radiation may cause the observed diffusion effects.

\section{REFERENCES}

1. Erley W. and Wagner H., Phys. Stat. Solid. (a), vol. 6, p. 543, (1971).

2. Churin V.A., Koriukin V.A., Vasilyev I.V., VANT, Series: Nuclear Reactor Physics, Issue 1, p. 59, (2015).

3. Kukharkin N.E., Ponomarev-Stepnoi N.N., Usov V.A. Space Nuclear Power Systems (ROMASHKA and ENISSEY Nuclear Reactors with Thermoelectric and Thermionic Energy Conversion)/Under the editorship of RAS Member N.N. Ponomarev-Stepnoi / IzdAT, Moscow, 146 p., (2008).

4. V.A. Stepanov, Technical Physics Journal, vol. 68, No. 8, p. 67, (1998).

5. Indenbom V.L., Technical Physics Journal Letters, vol. 5, Issue 8, p. 489, (1979).

6. Gieb M., Heieck J., Schule W., J. Nuc. Mat., vol. 225, p. 85, (1995).

7. Degaltsev Yu.G., Ponomarev-Stepnoi N.N., Kuznetsov V.F. Behavior of High-temperature Nuclear Fuel, Energoatomizdat, Moscow, (1987).

8. Pouchou J.I., Pichoir F. Basic expression of "PAP" computation for quantitative EPMA, Proc. of $11^{\text {th }}$ Intern. Congr. on X-Ray Optics and Microanalysis, Canada-London, p. 249, (1986).

9. Borovsky I.B., Gurov K.P., Marchukova I.D., Ugaste Yu.E., Interdiffusion Processes in Alloys, Nauka, Moscow, 360 p. (1973).

10. V.V. Uglov, Radiation Processes and Phenomena in Solids, Vysheishaya Shkola, Minsk 188 p. (2016). 